\title{
Discovery of higher-order quantum electrodynamics effect for the vacuum pair production
}

\section{W. Zha and Z. Tang ${ }^{1}$}

State Key Laboratory of Particle Detection and Electronics, University of Science and Technology of China, No. 96 JinZhai Road, Hefei City, Anhui Province, China

Department of Modern Physics, University of Science and Technology of China, No. 96 JinZhai Road, Hefei City, Anhui Province, China

E-mail: first@ustc.edu.cn, zbtang@ustc.edu.cn

ABSTRACT: The higher-order quantum electrodynamics (QED) effect for vacuum pair production has been searched without success since 1954. In this paper, we show that the combined world-wide data of lepton pair vacuum production is about $20 \%$ smaller than the latest lowest order QED calculation with a 5.2 sigma-level of significance and is consistent with the corresponding higher-order QED result. We claim the discovery of higher-order effect for the QED pair production, which settles the dust of previous debates for several decades. The verification of higher-order QED effect is a fundamental scientific problem, which is an important milestone towards the nonperturbative and nonlinear regime of QED vacuum.

Keywords: Heavy Ion Phenomenology

ArXiv EPrint: 2103.04605

\footnotetext{
${ }^{1}$ Corresponding author.
} 


\section{Contents}

1 Introduction 1

2 Theoretical setup $\quad 3$

2.1 Lowest order QED approach 3

$\begin{array}{ll}2.2 & \text { Higher-order correction to all orders }\end{array}$

$\begin{array}{lll}2.3 & \text { Equivalent photon approximation } & 7\end{array}$

$\begin{array}{lll}2.4 & \text { The trigger probability in ultra-peripheral collisions } & 8\end{array}$

$\begin{array}{llr}3 & \text { Results } & 10\end{array}$

4 Summary 13

\section{Introduction}

Quantum electrodynamics (QED) is a quantum field theory of the electromagnetic force [1-3], which describes the gauge invariant interaction of charged particles with photons. Due to the quantum fluctuations, the QED vacuum behaves like a polarizable medium, leading to novel quantum effects, such as Casimir effect [4], elastic photonphoton scattering $[5,6]$ and pair production from vacuum in extreme strong electromagnetic field [7]. The vacuum pair production is a process in which virtual dipole pair in the vacuum can be accelerated apart by the external field, emerging as real pair. In the laboratory, the vacuum pair production can be realized in the strong Coulomb field of relativistic heavy ion collisions [8]. The electromagnetic fields accompanied in these collisions are of order of [9]

$$
E_{\max } \simeq \frac{Z e \gamma}{b^{2}}
$$

where $Z e$ is the electric charge carried by the colliding ion, $b$ is the impact parameter, and $\gamma$ is the Lorentz factor of nuclei in center of mass frame. For gold-gold collisions at highest energy from the Relativistic Heavy Ion Collider (RHIC), with the values of $Z=79$, $b=15 \mathrm{fm}$ and $\gamma=108$, the maximum field strength is of $E_{\max }=5.3 \times 10^{16} \mathrm{~V} / \mathrm{cm}$. For the lead-lead collisions at the Large Hadron Collider (LHC), we get $E_{\max }=1.4 \times 10^{18} \mathrm{~V} / \mathrm{cm}$ with the values of $Z=82, b=15 \mathrm{fm}$ and $\gamma=2706$. The duration time of the strong field is very short, which makes the perturbative theory still appropriate for vacuum pair production. As illustrated in figure 1, the pair production can be treated perturbatively via the collision of two quasi-real light quanta from the strong Coulomb fields surrounding the heavy ions, which is very similar to the famous Breit-Wheeler process [10]. The theoretical investigations of vacuum pair production in heavy-ion collisions go back to the early days of QED. The lowest order (Born approximation) results were given by Landau and Lifshitz [11] and Racah [12] in the thirties of the past century. 


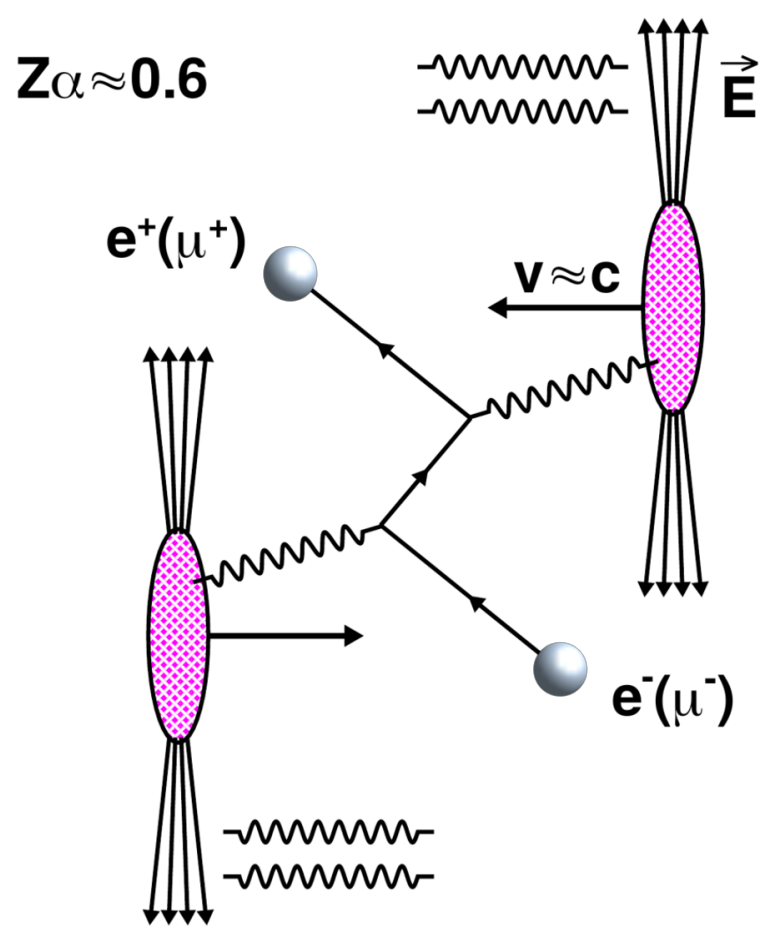

Figure 1. (Color online) An illustration of vacuum pair production process from the strong Coulomb field of fast moving nuclei in heavy ion collisions.

Due to the large charge carried by the heavy ion, the effect coupling $Z \alpha(\sim 0.6$ for gold and lead) for the production process is close to 1 . This suggests significant higher-order effect, which links the crossover from perturbative to nonperturbative regime of QED. This is analogous to the case of Quantum Chromodynamics (QCD) $[13,14]$ from high energy to low energy area. In 1954, the pioneer studies $[15,16]$ for higher-order effect of QED were made by Bethe, Maximon and Davies in a similar process - Bethe-Heitler process [17] (the photoproduction of electron-positron pair in the nuclear Coulomb field). Higher-order effect was treated using the Sommerfeld-Maue wave function, which is appropriate solution of the Dirac equation at high energy. This approach takes higher-order effect into account to all orders and can be related to the usual Feynman graph technique [18]. A sizeable negative correction was found to the Bethe-Heitler formula. Back to the case of vacuum pair production in relativistic heavy ion collisions, the correction should be stronger, since the projectile quasi-real photon is also attached to the heavy ion in contrast to the BetheHeitler process.

The theoretical, as well as, experimental investigations of higher-order effect for vacuum pair production in heavy ion collisions were spurred in the late 20th century by the relativistic heavy ion facilities Alternating Gradient Synchrotron (AGS) at Brookhaven, the Super Proton Synchrotron (SPS) at the European Organization for Nuclear Research (CERN) and more recently RHIC at Brookhaven and LHC at CERN. The higher-order 
correction in heavy-ion collisions can be studied by the regularization to the photon propagator in the 'Sommerfeld-Maue' type approach. The authors of [19, 20] merely regularized the photon propagator at large distance, in which the higher-order correction represents itself as a common phase shift. This led to the absence of higher correction to total cross section for vacuum pair production. With the same regularization, the study of $[21,22]$ indicated that the higher-order effect could only be observed in the differential cross section as a function of impact parameter. This is in obvious contradiction to the special case of the Bethe-Maximon correction to the Bethe-Heitler formula. To solve the inconsistency, Lee and Milstein [23] constructed an appropriate regularization by introducing a screening of the Coulomb potential, which resulted in a large negative correction to the total cross section. However, with the multiple pair production taken into account [24], the higher-order effect for total cross section became small. Following this strategy, the hint of higher-order effect $[25,26]$ had been observed in comparison with the RHIC measurements. Over the decades, various experimental measurements of lepton pair production [27-34] has been made in ultra-peripheral collisions (UPC) at RHIC and LHC. In these measurements, due to the limited kinetic space, the multiple production of vacuum pair should be negligible. Surprisingly, all the measurements are found to be in good agreement with the calculations of equivalent photon approximation approach, which are identical to the lowest order QED results for cross section estimation. The searching for higher-order effect seems to be hopeless, just as the statement in ref. [9]: "In April 1990 a workshop took place in Brookhaven with the title 'Can RHIC be used to test QED?'. We think that after about 17 years the answer to this question is 'no'." This is very puzzling: if the higher-order effect is not there, how could we expect the nonperturbative vacuum pair production from Schwinger mechanism? And exploring the nonperturbative feature of QED is one of the primary goals for the planning ultra strong laser facilities in the not-so-far future.

The paper is organized as follows. In section 2, we introduce the theoretical setup employed to calculate vacuum pair production in ultra-peripheral heavy-ion collisions (UPC). In section 3, we present the latest lowest order QED calculations for lepton pair production in heavy-ion collisions and find that the result is about 5.2 standard deviations larger than the combined world-wide data. Furthermore, the higher-order QED correction to all orders is carried out, which reduces the lowest order results sizably $(\sim 20 \%)$. Taken the higher-order effect into account, the corresponding result is consistent with data, which claims the discovery of higher-order effect for the QED pair production, and settles the dust of previous debates for several decades. We also discuss the missing part in the old lowest order calculations, which prevents us from the observation of higher-order QED effect. Finally, the paper is summarized in section 4.

\section{Theoretical setup}

\subsection{Lowest order QED approach}

The total cross section of vacuum pair production in lowest order due to the Coulomb fields of two colliding nuclei has been carried out for the first time by Landau and Lifshitz [11] 
in 1934. However, the experimental measurements are usually performed in limited kinematics phase due to the limited acceptance covered by the detector system, which calls for differential theoretical calculations. We treat the electromagnetic fields of the heavy ions as external fields and employ "straight line approximation" since the deflection angle due to the Coulomb scattering is very small. The electromagnetic fields of the colliding nuclei are then given by

$$
A_{\mu}^{(1,2)}(q)=-2 \pi Z e \mu_{\mu}^{(1,2)} \delta\left(q \mu^{(1,2)}\right) \frac{f\left(q^{2}\right)}{q^{2}} \exp ( \pm \mathrm{iqb} / 2),
$$

where $Z$ is the electric charge carried by ions, $\mu^{(1,2)}$ is the four-velocity of nuclei 1 and $2, b$ is the impact parameter of collisions, and the form factor $f\left(q^{2}\right)$ is Fourier transform of the charge distribution in nucleus. In the calculation, we employ the Woods-Saxon form [35] to model the charge distribution of nucleus in spherical coordinates:

$$
\rho_{A}(r)=\frac{\rho^{0}}{1+\exp \left[\left(r-R_{\mathrm{WS}}\right) / d\right]},
$$

where the radius $R_{W S}(\mathrm{Au}: 6.38 \mathrm{fm}, \mathrm{Pb}: 6.62 \mathrm{fm})$ and skin depth $d(\mathrm{Au}: 0.535 \mathrm{fm}, \mathrm{Pb}$ : $0.546 \mathrm{fm})$ are based on fits to electron-scattering data [36] and $\rho^{0}$ is the normalization factor. For $p+p(\bar{p})$ collisions, the standard dipole form factor of proton (anti-proton) is utilized with the electric charge radius determined $\left(\left\langle r_{E}\right\rangle=0.879 \mathrm{fm}\right)$ by the latest electronproton scattering data [37]. For symmetric collisions, $Z$ and $f\left(q^{2}\right)$ are the same for both nuclei. In center of mass frame, the four-velocity of nuclei can be written as

$$
\mu^{(1,2)}(q)=\gamma(1,0,0, \pm \beta)=: \gamma w^{(1,2)}
$$

where $\gamma$ and $\beta$ are the Lorentz factor and velocity of colliding nuclei, respectively. Following the derivations of refs. [38, 39], with the direct and cross Feynman diagrams, the matrix element for the creation of a lepton pair is given by

$$
M=\bar{\mu} \hat{M} v
$$

with

$$
\begin{aligned}
\hat{M}= & -i e^{2} \int \frac{d^{4} q_{1}}{(2 \pi)^{4}} A^{(1)}\left(q_{1}\right) \frac{\not p_{-}-\not q_{1}+m}{\left(p_{-}-q_{1}\right)^{2}-m^{2}} A^{(2)}\left(p_{+}+p_{-}-q_{1}\right) \\
& -i e^{2} \int \frac{d^{4} q_{1}}{(2 \pi)^{4}} A^{(2)}\left(p_{+}+p_{-}-q_{1}\right) \frac{\not_{1}-\not p_{+}+m}{\left(q_{1}-p_{+}\right)^{2}-m^{2}} \mathcal{A}^{(1)}\left(q_{1}\right) \\
= & -i\left(\frac{Z e^{2}}{2 \pi}\right)^{2} \frac{1}{2 \beta} \int d^{2} q_{1 \perp} \frac{1}{q_{1}^{2}} \frac{1}{\left(p_{+}+p_{-}-q_{1}\right)^{2}} \exp \left(\mathrm{iq}_{1 \perp} \mathrm{b}\right) \\
& \left\{\frac{\psi^{(1)}\left(\not p_{-}-\not q_{1}+m\right) \psi^{(2)}}{\left[\left(p_{-}-q_{1}\right)^{2}-m^{2}\right]}+\frac{\psi^{(2)}\left(q_{1}-\not p_{+}+m\right) \psi^{(1)}}{\left[\left(q_{1}-p_{+}\right)^{2}-m^{2}\right]}\right\},
\end{aligned}
$$

where the longitudinal components of $q_{1}$ are given by $q_{10}=\frac{1}{2}\left[\left(\epsilon_{+}+\epsilon_{-}\right)+\beta\left(p_{+z}+p_{-z}\right)\right]$, $q_{1 z}=q_{10} / \beta, \epsilon_{+}$and $\epsilon_{-}$are the energies of the produced leptons, and $m$ is the mass of 
lepton. One can get the differential probability by taking the absolute value squared of $M$ and summing over the spin indices as

$$
\begin{aligned}
P\left(p_{+}, p_{-}, b\right)=\sum_{s}|M|^{2}= & \frac{4}{\beta^{2}} \int d^{2} q_{1 \perp} d^{2} q_{\perp} \exp \left(\mathrm{iq}_{\perp} \mathrm{b}\right) \\
& \times f\left(N_{0}\right) f\left(N_{1}\right) f\left(N_{3}\right) f\left(N_{4}\right) F\left(N_{0}\right) F\left(N_{1}\right) F\left(N_{3}\right) F\left(N_{4}\right) \\
& \times \operatorname{Tr}\left\{\left(\not p_{-}+m\right)\left[N_{2 D}^{-1} \psi_{1}\left(\not p_{-}-\not q_{1}+m\right) \psi_{2}+N_{2 X}^{-1} \psi_{2}\left(\not q_{1}-\not p_{+}+m\right) \psi_{1}\right]\right. \\
& \left.\left(\not p_{+}-m\right)\left[N_{5 D}^{-1} \psi_{2}\left(\not p_{-}-\not q_{1}-\not q+m\right) \psi_{1}+N_{5 X}^{-1} \psi_{1}\left(\not q_{1}+\not q_{-}-\not p_{+}+m\right) \psi_{2}\right]\right\},
\end{aligned}
$$

with

$$
\begin{aligned}
N_{0} & =-q_{1}^{2}, N_{1}=-\left[q_{1}-\left(p_{+}+p_{-}\right)\right]^{2}, \\
N_{3} & =-\left(q_{1}+q\right)^{2}, N_{4}=-\left[q+\left(q_{1}-p_{+}-p_{-}\right)\right]^{2}, \\
N_{2 D} & =-\left(q_{1}-p_{-}\right)^{2}+m^{2}, \\
N_{2 X} & =-\left(q_{1}-p_{+}\right)^{2}+m^{2}, \\
N_{5 D} & =-\left(q_{1}+q-p_{-}\right)^{2}+m^{2}, \\
N_{5 X} & =-\left(q_{1}+q-p_{+}\right)^{2}+m^{2} .
\end{aligned}
$$

The photon propagators $F\left(N_{k}\right)$ attached to the Coulomb fields of heavy ions can be written as

$$
F\left(N_{k}\right)=\frac{Z \alpha}{N_{k}}=\frac{Z \alpha}{\frac{w_{k}^{2}}{\gamma^{2}}+p_{T k}^{2}}, k=0,1,3,4,
$$

where $\alpha\left(\sim \frac{1}{137}\right)$ is the fine structure constant, and $w_{k}$ and $p_{T k}$ are the energies and transverse momenta of the photons, respectively. For the total cross section, we integrate over $p_{+}, p_{-}$, and $b$ to get

$$
\sigma=\int d^{2} b \frac{d^{3} p_{+} d^{3} p_{-}}{(2 \pi)^{6} 2 \epsilon_{+} 2 \epsilon_{-}} P\left(p_{+}, p_{-}, b\right)
$$

In the calculation of $P\left(p_{+}, p_{-}, b\right)$, the trace is treated with the help of the Mathematica package FeynCalc [40]. The multiple dimension integration is done with the help of the Monte Carlo (MC) integration routine VEGAS [41].

In the lowest order QED calculations, the internal photon radiation is not considered, which leads to the absence of tail in the transverse momentum distributions of the produced vacuum pairs. In ref. [42], S.R. Klein etal. estimated the QED showering effect in a Sudakov formalism, which qualitatively described the notable tail in acoplanarity distributions observed by ATLAS [43]. To include this effect, the lowest-order QED calculations are provided as input into Pythia8 (version 8.305 [44, 45]) for QED showering. The showering process would not affect the overall cross section, however can lead to a fraction of the events migrating in and out of the selected fiducial region used in the experimental measurements. For a fair comparison with experimental data, this effect should be considered to correctly estimated the acceptance in the measured kinematic region. 


\subsection{Higher-order correction to all orders}

It was pointed out by Ivanov, Schiller, and Serbo [46] that the higher-order effect (or Coulomb correction) of lepton pair production in heavy-ion collisions was analogous to the well know Bethe-Heitler process on a heavy target [17]. The higher-order effect in BetheHeitler process was first studied by Bethe, Maximon and Davies [15, 16]. They summed up the higher-order term using Sommerfeld-Maue wave function, which is approximate solution of the Dirac equation in the nuclear Coulomb field valid for high energies and small scattering angles; and found a negative higher-order correction proportional to $Z^{2}$. It is very interesting to see that the standard method of summing up all Feynman graphs gives identical results [18], which establishes the equivalence between the calculations using Sommerfeld-Maue wave function and the standard higher-order QED approach. This is very important for our study of high-order effect in heavy ion collisions: there are again the Feynman diagram approach and the 'Sommerfeld-Maue type' of approach.

The 'Sommerfeld-Maue type' approach in heavy-ion collisions results from an exact solution of the time dependent Dirac equation in the ultra-relativistic limit [19]. In this approach, the high-order correction is present by the regularization to the photon propagator. The photon propagator $F(k)$ describes the interaction with the Coulomb field of one of the nuclei. It is proportional to the lepton eikonal scattering amplitude for one of the potentials $V\left(r_{\perp}, z\right)$ :

$$
F(k)=\int d^{2} r_{\perp} \exp \left(-\mathrm{ikr}_{\perp}\right)\left\{\exp \left[-\mathrm{i} \chi\left(\mathrm{r}_{\perp}\right)\right]-1\right\}
$$

where

$$
\chi\left(r_{\perp}\right)=\int_{-\infty}^{+\infty} d z V\left(r_{\perp}, z\right)
$$

For an unscreened Coulomb potential $V\left(r_{\perp}, z\right)=-Z \alpha / \sqrt{r_{\perp}^{2}+z^{2}}$, the photon propagator can be written as

$$
F(k)=\int d^{2} r_{\perp} \exp \left(-\mathrm{ikr}_{\perp}\right)\left[\exp \left(-2 \mathrm{iz} \alpha \ln r_{\perp}\right)-1\right]
$$

If one merely regularizes $F(k)$ at large $r$, it gives [19, 20]

$$
F(k)=\frac{4 \pi \alpha Z}{k^{2-2 i \alpha Z}} .
$$

And a lower $k$ cutoff at some $\omega / \gamma$ has to be put in the photon propagator by hand to obtain dependence on the collision energy and to agree with the known perturbative result, which modifies $F(k)$ to

$$
F(k)=\frac{4 \pi \alpha Z}{\left(k^{2}+\omega^{2} / \gamma^{2}\right)^{1-i \alpha Z}} .
$$

Then the higher-order effect is introduced by a common phase shift in the denominator of eq. (2.14), which means that the higher-order correction would be absent for the total cross section. This is contradicted with the physical picture extracted from Bethe-Maximon 
theory. In order to solve the contradiction and construct an appropriate regularization, Lee and Milstein [23] introduce a screening of the Coulomb potential as

$$
V\left(r_{\perp}, z\right)=\frac{-Z \alpha \exp \left(-\sqrt{\mathrm{r}_{\perp}^{2}+\mathrm{z}^{2}} \omega / \gamma\right)}{\sqrt{r_{\perp}^{2}+z^{2}}} .
$$

Then the integral eq. (2.11) can be carried out to obtain

$$
\chi\left(r_{\perp}\right)=-2 Z \alpha K_{0}\left(r_{\perp} \omega / \gamma\right),
$$

and the photon propagator is modified to

$$
F(k)=\frac{1}{2} \int d r_{\perp} r_{\perp} J_{0}\left(k r_{\perp}\right)\left\{\exp \left[2 \mathrm{iZ} \alpha \mathrm{K}_{0}\left(\mathrm{r}_{\perp} \omega / \gamma\right)\right]-1\right\} .
$$

In the limit as $Z \alpha \rightarrow 0$, eq. (2.17) goes to the familiar perturbation theory form

$$
F^{0}(k)=\frac{4 \pi i Z \alpha}{k^{2}+\omega^{2} / \gamma^{2}} .
$$

In the calculation, we employ eq. (2.17) to estimate the higher-order correction.

\subsection{Equivalent photon approximation}

As revealed in eq. (2.6), there are apparent numerical difficulties in evaluating the multiple dimension integration due to the oscillating factor $e^{i \vec{q} \cdot \vec{b}}$. A simpler model, EPA approach, is widely used, which is identical to the lowest order QED calculation for the cross section estimation. Since most the experimental results are compared to EPA calculations to test the validity of lowest order QED, we briefly introduce it here. In the approach, the vacuum pair production in heavy-ion collisions can be factorized into a semiclassical and quantum part. The semiclassical part deals with the distribution of quasi-real photons induced by the colliding ions, while the quantum part handles the interactions of photon-photon. It gives:

$$
\begin{aligned}
& \sigma\left(A+A \rightarrow A+A+l^{+} l^{-}\right) \\
& =\int d \omega_{1} d \omega_{2} n\left(\omega_{1}\right) n\left(\omega_{2}\right) \sigma\left(\gamma \gamma \rightarrow l^{+} l^{-}\right),
\end{aligned}
$$

where $\omega_{1}$ and $\omega_{2}$ are the photon energies from the two colliding beams, and $\sigma\left(\gamma \gamma \rightarrow l^{+} l^{-}\right)$ is the photon-photon reaction cross-section for lepton pair. The photon flux induced by the heavy ions can be modeled using the Weizsäcker-Williams method [47]:

$$
\begin{aligned}
n\left(\omega, r_{\perp}\right) & =\frac{4 Z^{2} \alpha}{\omega}\left|\int \frac{\vec{q}_{\perp}}{(2 \pi)^{2}} \vec{q}_{\perp} \frac{f(\vec{q})}{q^{2}} e^{i \vec{q}_{\perp} \cdot r_{\perp}}\right|^{2} \\
\vec{q} & =\left(\vec{q}_{\perp}, \frac{\omega}{\gamma}\right)
\end{aligned}
$$

where $n\left(\omega, r_{\perp}\right)$ is the flux of photons with energy $\omega$ at distance $r_{\perp}$ from the center of nucleus. For the point-like charge distribution, the photon flux is given by the simple formula

$$
n\left(\omega, r_{\perp}\right)=\frac{Z^{2} \alpha}{\pi^{2} \omega r_{\perp}^{2}} x^{2} K_{1}^{2}(x), x=\omega r_{\perp} / \gamma
$$


The elementary cross-section to produce a lepton pair with lepton mass $m$ and pair invariant mass $W$ can be determined by the Breit-Wheeler formula [10].

$$
\begin{aligned}
\sigma\left(\gamma \gamma \rightarrow l^{+} l^{-}\right)=\frac{4 \pi \alpha^{2}}{W^{2}} & {\left[\left(2+\frac{8 m^{2}}{W^{2}}-\frac{16 m^{4}}{W^{4}}\right) \ln \left(\frac{W+\sqrt{W^{2}-4 m^{2}}}{2 m}\right)\right.} \\
& \left.-\sqrt{1-\frac{4 m^{2}}{W^{2}}}\left(1+\frac{4 m^{2}}{W^{2}}\right)\right]
\end{aligned}
$$

As discussed in our previous work [48], the standard and generalized EPA calculations are powerless to reproduce the differential transverse momentum spectra of lepton pairs, however, for the total cross section versus impact parameter, they give the same results as the lowest order QED calculations, which establishes the equivalence between EPA approaches and the lowest order QED on total cross section estimation. In reality, due to the limited acceptance covered by the detector system, the experimental measurements on cross section are usually made in limited transverse momentum region of lepton pairs, which weakens the accuracy of EPA calculations. Fortunately, the lepton pairs are mostly produced at very low transverse momentum, the effect originated from the incorrect transverse momentum spectra of lepton pairs should be small.

\subsection{The trigger probability in ultra-peripheral collisions}

At small impact parameters, the vacuum pair production could be accompanied by hadronic processes, which contaminates the exclusive process with additional hadrons. To avoid needing to disentangle these two processes, the ultra-peripheral events are triggered without any nuclear overlap. In heavy-ion collisions, the probability of having no hadronic interaction can be estimated by Glauber model [35]. According to the optical Glauber model, the mean number of projectile nucleons that interact at least once in $\mathrm{A}+\mathrm{A}$ collisions with impact parameter $b$ is:

$$
m_{H}(b)=\int d^{2} r_{\perp} T_{A}\left(r_{\perp}-b\right)\left\{1-\exp \left[-\sigma_{\mathrm{NN}} \mathrm{T}_{\mathrm{A}}\left(\mathrm{r}_{\perp}\right)\right]\right\},
$$

where $T_{A}\left(r_{\perp}\right)$ is the nuclear thickness function determined from the nuclear density distribution, and $\sigma_{\mathrm{NN}}$ is the total nucleon-nucleon cross section. Here, the nuclear density distribution can be written as:

$$
\rho_{N}(r)=\frac{Z}{A} \rho_{p}(r)+\frac{N}{A} \rho_{n}(r)
$$

where $\rho_{p}(r)$ and $\rho_{n}(r)$ are the proton and neutron density distributions, respectively. For symmetric $(N=Z)$ nuclei, it is expected that the proton and neutron density distributions have similar shapes, which could be parameterized as eq. (2.2). In heavy or unstable neutron-rich nuclei $(N \gg Z)$, the excess neutrons are pushed out against surface tension forming a neutron skin, which is defined as the difference between the neutron and proton root-mean-square radii: $\Delta r_{n p}=\left\langle r_{n}^{2}\right\rangle^{1 / 2}-\left\langle r_{p}^{2}\right\rangle^{1 / 2}$. Therefore, in neutron-rich nuclei, the neutron distribution is expected to have a larger radius than that of proton distribution, but with a similar skin depth parameter. We employ the parameterization of neutron skin 
thickness $\Delta r_{n p}$ in [49] (Au: $0.26 \mathrm{fm}, \mathrm{Pb}: 0.28 \mathrm{fm}$ ) in the calculation, which is consistent with the recent experimental measurement [50] for lead. The total nucleon-nucleon cross section is extracted from the recommendation of Particle Data Group [51] (0.2 TeV: $42 \mathrm{mb}$, $2.76 \mathrm{TeV}: 62 \mathrm{mb}$, and $5.02 \mathrm{TeV}: 68 \mathrm{mb}$ ). Then, the probability with no hadronic interaction in heavy-ion collisions is:

$$
P_{H}(b)=\exp \left[-\mathrm{m}_{\mathrm{H}}(\mathrm{b})\right] .
$$

For $p+p(\bar{p})$ collisions, the probability with no inelastic collision in impact parameter space can be modeled by [52]

$$
P_{\mathrm{H}}=\left|1-\exp \left(-\mathrm{b}^{2} /(2 \mathrm{~B})\right)\right|^{2},
$$

where $B$ is determined by the experimental measurements [51, 53].

The vacuum pair production could also be accompanied by Coulomb dissociation of nuclei, which leads to the presence of forward neutrons. The forward neutron multiplicity can be used to select different impact parameter ranges in ultra-peripheral collisions. The electromagnetic dissociation probability of nuclei with different neutron tag can be estimated by EPA method. The Coulomb excitation of an ultra-relativistic nucleus can be factorized into two parts [54]: the distribution of quasi-real photons induced by the colliding nuclei, and the appropriate photon-absorption cross section of nuclei. The lowest-order probability for an excitation to the state which emits at least one neutron $\left(X_{n}\right)$ is

$$
m_{X n}(b)=\int d k n(b, E) \sigma_{\gamma A \rightarrow A^{*}}(E),
$$

where $E$ is the photon energy, $n(b, E)$ is the flux of photons with energy $E$ at distance $b$ from the center of nucleus, and $\sigma_{\gamma A \rightarrow A^{*}}(E)$ is the photoexcitation cross section with incident energy $E$. The photon flux generated by the nucleus can be modeled by the Weizsäcker-Williams method, shown as eq. (2.20) and (2.21). The photoexcitation cross section $\sigma_{\gamma A \rightarrow A^{*}}(E)$ can be determined from the experimental measurements [55-61]. In high energy collisions, for example at RHIC top energy or LHC energies, $m_{X n}(b)$ would exceed 1 at small impact parameter, which can not be interpreted as a probability. In ref. [62], $m_{X n}(b)$ was treated as the mean number of excitations with a Poisson distribution. Then the probability of zero neutron emission is equal to

$$
P_{0 n}(b)=e^{-m_{X n}(b)},
$$

and the probability for at least one neutron emission is

$$
P_{X n}(b)=1.0-e^{-m_{X n}(b)} .
$$

The probability of mutual electromagnetic dissociation, under the assumption of independent nuclear break-up, can be factorized as the product of the dissociation probability of each nucleus:

$$
\begin{aligned}
P_{X n X n}(b) & =\left(1.0-e^{-m_{X n}(b)}\right)^{2}, \\
P_{0 n X n}(b) & =2\left(1.0-e^{-m_{X n}(b)}\right) e^{-m_{X n}(b)}, \\
P_{* n X n}(b) & =1.0-e^{-2 m_{X n}(b)} \\
P_{0 n 0 n}(b) & =e^{-2 m_{X n}(b)}
\end{aligned}
$$

where $* n$ denotes the absence of requirement on neutron emission. 
Hereinbefore, the Coulomb dissociation of nuclei and the production of vacuum pair are assumed as independent processes. In reality, it is also possible that the production of vacuum pair leads to the dissociation of nuclei: the nuclei can be excited and dissociated due to the emission of the photon, which is known as incoherent photon emission. In experiment, it is hard to distinguish the vacuum pair production with nuclear break-up from their incoherent production. The incoherent contribution can be estimated by the EPA approach. Following ref. [63], the equivalent photon flux due to the incoherent emission can be written as

$$
n^{\mathrm{incoh}}(\omega, b)=\int d^{2} r_{\perp} T_{Z}\left(r_{\perp}\right) n^{\text {proton }}\left(\omega, b+r_{\perp}\right)-n(\omega, b) / Z,
$$

where $n(\omega, b)$ is the usual equivalent photon flux for a given nucleus, see eq. (2.20) and (2.21), $n^{\text {proton }}(\omega, b)$ denotes the equivalent photon spectrum of proton, and $T_{Z}\left(r_{\perp}\right)$ is the proton thickness function of nucleus. As shown in eq. (2.31), the incoherent contribution is proportional to $Z$, whereas the coherent part is proportional to $Z^{2}$. In heavy-ion collisions, one expects the coherent contribution to be dominant. For semi-coherent production of vacuum pair (one photon from incoherent emission, the other from coherent process), the cross section can be estimated by replacing one of the coherent photon fluxes $n(\omega, r)$ by the incoherent one $n^{\text {incoh }}(\omega, r)$ in eq. (2.19). For incoherent production, both the coherent photon fluxes should be displaced by the incoherent ones.

Could the nuclei be dissociated by the lepton pair, when the pair production occurs within one of the nuclei? For the lepton pair produced within one of the nuclei, they would travel through the nuclei with time scale about $R_{A} / \gamma c(\sim 0.06 \mathrm{fm} / \mathrm{c}$ for RHIC, and $\sim 0.006 \mathrm{fm} / \mathrm{c}$ for LHC) after production. At such small time after production, the distance between the lepton pair is very small, so the lepton pair can be viewed as a whole with zero electric charge. Thus, the lepton pair would have negligible electromagnetic interaction with the nuclei, which would barely cause the nuclei to dissociate. In the calculation, we ignore the probability of nuclear dissociation induced by the lepton pair production within one of the nuclei.

\section{Results}

Various exclusive experimental measurements [64-67] for lepton pair production in $p+p(\bar{p})$ collisions have been made, despite of the tiny cross section due to $Z=1$. The coupling constant in these collisions is in perturbative limit $(Z \alpha \rightarrow 0)$, which provides excellent baseline to test the validity of lowest order QED. Figure 2 shows comparison of the ratios of the measured cross sections from world-wide experiments [64-67] to the lowest order QED calculations for lepton pair production in $p+p(\bar{p})$ collisions. The error bars represent the total uncertainties for the experimental measurements, in which the statistical and systematic errors are added in quadrature. The theoretical uncertainties are estimated by varying the parameters in the proton form factor and the UPC trigger probability, which are found to be less than $2 \%$. The yellow bands in the figure represent the uncertainties for lowest order QED calculations. As shown in figure, the lowest order QED calculations describe the world-wide measurements very well. The world-wide results are combined with 


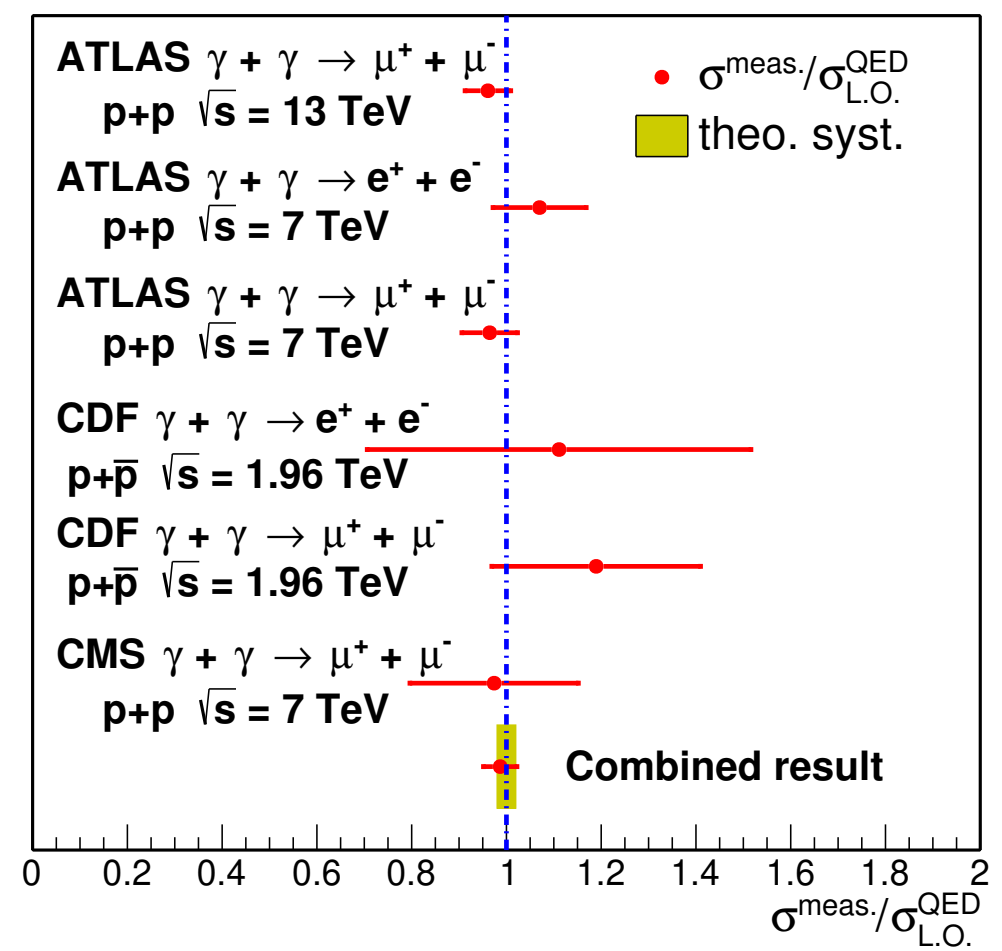

Figure 2. (Color online) Comparison of the ratios of the measured cross sections from world-wide experiments [64-67] to the lowest order QED calculations for lepton pair production in $p+p(\bar{p})$ collisions. The error bars represent the total uncertainties for the experimental measurements, which include the statistical and systematic errors. The yellow bands denote the uncertainties for lowest order QED calculations.

unequal weights determined by the errors to improve the precision of measurement. In the combination of results, the uncertainties from luminosity determination at the same collider have been considered to be fully correlated and the systematic uncertainties associated with detector response are assumed to be completely correlated at the same Collaboration. The uncertainties from theoretical calculations are also combined with the assumption of being fully correlated. The combined result is consistent with the lowest order QED calculation within one standard deviation.

The comparison of the ratios of the measured cross sections from world-wide experiments [29-34] and the predicted higher-order QED results to the lowest order QED calculations for lepton pair production in heavy ion collisions is shown in figure 3. In the calculation, optical Glauber model [35] is employed to determine the UPC trigger probability of heavy ion collision in impact parameter space. The theoretical uncertainties of vacuum pair production are estimated by varying the parameters in the Woods-Saxon distributions of heavy nuclei, which simultaneously changes the form factor of heavy nuclei and the UPC trigger probability in impact parameter space. The uncertainty originated from this is less than $2.5 \%$. Some of the experimental measurements are performed with mutual Coulomb dissociation of the colliding nuclei. The calculations of mutual Coulomb dissociation probabilities are introduced in section 2.4. The uncertainties associated with 


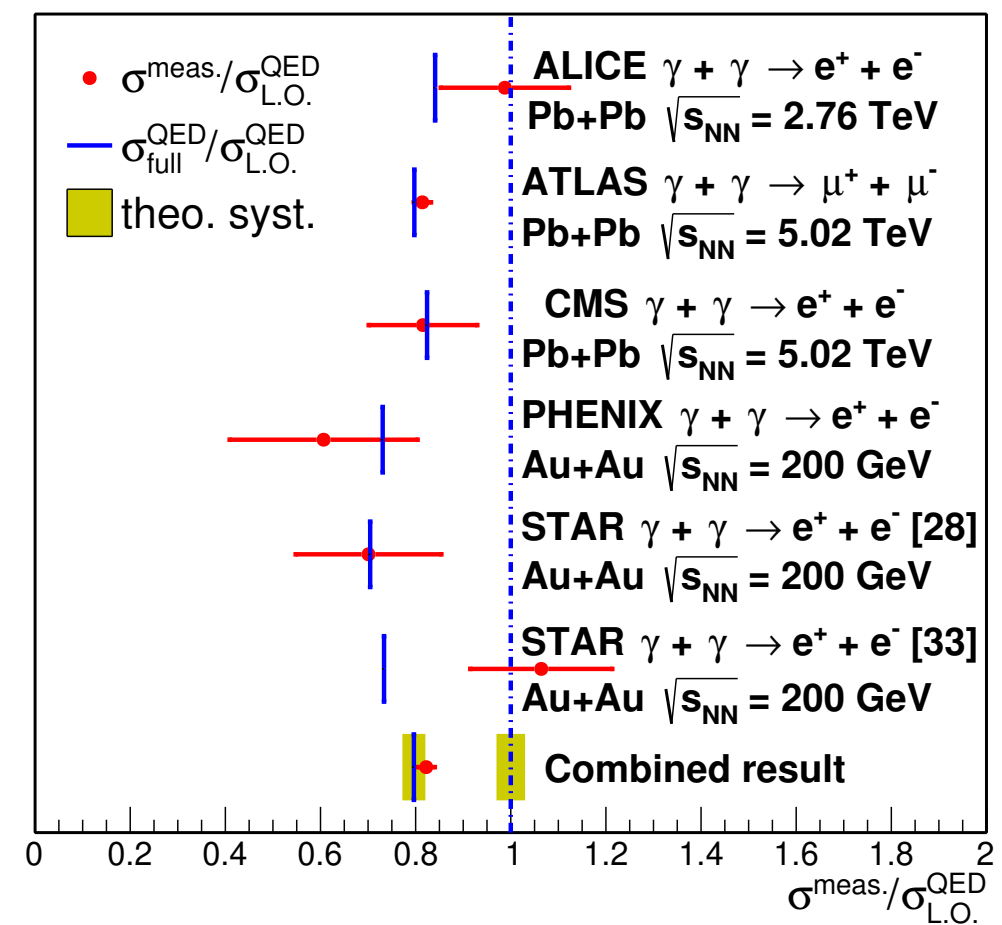

Figure 3. (Color online) Comparison of the ratios of the measured cross sections from worldwide experiments [29-34] and the predicted higher-order QED results to the lowest order QED calculations for lepton pair production in $A+A$ collisions. The error bars represent the total uncertainties for the experimental measurements, which include the statistical and systematic errors. The yellow bands denote the uncertainties for QED calculations.

Coulomb dissociation for $X n X n$ and $* n X n$ (at least one neutron emission) configurations are about $11 \%$ and $6 \%$ respectively, which mainly result from the experimental errors for the photonuclear cross sections. The contribution of vacuum pair production related to incoherent photon emission is estimated by EPA approach, see section 2.4, which is taken as an additional source of theoretical uncertainty. The uncertainties from incoherent photon emission for $X n X n, * n X n$, and $* n * n$ (no requirement on neutron emission) configurations are about $6.5 \%, 3.4 \%$, and $1.5 \%$, respectively. The combined theoretical uncertainty associated with the combined world-wide experimental data is about $3 \%$. As demonstrated in the figure, except for the STAR [34] and ALICE [31] results, the measurements are systematically smaller than the lowest order QED predictions and the QED results with higher-order correction describe the data very well. Considering the large experimental and theoretical uncertainties for the STAR (experimental uncertainty: 14\%, theoretical uncertainty: 13\%) and ALICE (experimental uncertainty: 14\%, theoretical uncertainty: $3 \%)$ measurements, they are both consistent with the lowest order and higher-order QED predictions. The combined data is 5.2 standard deviations smaller than the lowest order calculation and is consistent with the higher-order result within one standard deviation, which claims the discovery of higher-order effect for the QED pair production in heavy ion collisions. 
As shown in figure 3, the higher-order corrections are significant $(\sim 20 \%)$. This raises a puzzle: why the higher-order effect is not observed previously? Most the experimental measurements are compared to a industry standard model - STARLight [68], which incorporates the equivalent photon approximation (EPA) approach to calculate the lepton pair production. The EPA approach is identical to the lowest order QED calculation for cross section estimation. In STARLight model, it treats the nucleus as a point-like charge for evaluating the photon flux. To avoid the singularities in the spatial distribution of photon flux, the pair production within the geometrical radius of the nucleus is ignored. However, according to our study, the production within the geometrical radius of the nucleus is not negligible. As an illustration, for the dimuon production in $\mathrm{Pb}+\mathrm{Pb}$ collisions at $\sqrt{s_{\mathrm{NN}}}=5.02 \mathrm{TeV}$ with the fiducial acceptance of ATLAS $\left(p_{T \mu}>5 \mathrm{GeV} / \mathrm{c},\left|\eta_{\mu}\right|<2.4\right)$, the fraction of production within the nuclei is about $28 \%$. Coincidentally, the higher-order correction to the lowest QED result is about $20 \%$, which is comparable to the fraction of production ignored in STARLight model. The two missing parts (the production within the nuclei and the higher-order correction) compensate with each other, which makes the STARLight model effective to describe world-wide data. This prevents us from the observation of higher-order effect.

\section{Summary}

We report the lowest-order QED calculations for lepton pair production both in protonproton (anti-proton) and heavy ion collisions. The lowest-order predictions describe the world-wide measurements in $p+p(\bar{p})$ collisions $(Z \alpha \rightarrow 0)$ very well, however, overestimate the production in heavy ion collision $(Z \alpha \sim 0.6)$ by about $20 \%$ with a 5.2 sigma-level of significance. The corresponding higher-order QED results can reproduce the world-wide measurements within one standard deviation. These findings lend credence to claim the discovery of higher-order effect for the QED pair production under the strongest electromagnetic field in laboratory, which have waited more than half a century for verification and pave the way for future tests of QED in the unexplored nonperturbative regimes. Furthermore, by colliding different species of nuclei, the coupling constant for the QED pair production can be varied to investigate the higher-order effect towards the nonperturbative regime, which provides a nice reference for the study of QCD from perturbative to nonperturbative area.

\section{Acknowledgments}

We thank Prof. Zhangbu Xu, Prof. Lijuan Ruan, Prof. Spencer Klein and Dr. Daniel Brandenburg for useful discussions. This work was funded by the National Natural Science Foundation of China under Grant Nos. 11775213 and 11720101001, and MOST under Grant No. 2018YFE0104900. Wangmei Zha is supported by "USTC Research Funds of the Double First-Class Initiative". 
Open Access. This article is distributed under the terms of the Creative Commons Attribution License (CC-BY 4.0), which permits any use, distribution and reproduction in any medium, provided the original author(s) and source are credited.

\section{References}

[1] J.S. Schwinger, Quantum electrodynamics. III: The electromagnetic properties of the electron: Radiative corrections to scattering, Phys. Rev. 76 (1949) 790 [INSPIRE].

[2] R.P. Feynman, The Theory of positrons, Phys. Rev. 76 (1949) 749 [inSPIRE].

[3] R.P. Feynman, Space - time approach to quantum electrodynamics, Phys. Rev. 76 (1949) 769 [INSPIRE].

[4] H. Casimir, On the Attraction Between Two Perfectly Conducting Plates, Indag. Math. 10 (1948) 261 [INSPIRE].

[5] ATLAS collaboration, Evidence for light-by-light scattering in heavy-ion collisions with the ATLAS detector at the LHC, Nature Phys. 13 (2017) 852.

[6] ATLAS collaboration, Observation of light-by-light scattering in ultraperipheral $\mathrm{Pb}+\mathrm{Pb}$ collisions with the ATLAS detector, Phys. Rev. Lett. 123 (2019) 052001 [arXiv:1904.03536] [INSPIRE].

[7] J.S. Schwinger, On gauge invariance and vacuum polarization, Phys. Rev. 82 (1951) 664 [INSPIRE].

[8] R. Ruffini, G. Vereshchagin and S.-S. Xue, Electron-positron pairs in physics and astrophysics: from heavy nuclei to black holes, Phys. Rept. 487 (2010) 1 [arXiv:0910.0974] [INSPIRE].

[9] G. Baur, K. Hencken and D. Trautmann, Electron-Positron Pair Production in Relativistic Heavy Ion Collisions, Phys. Rept. 453 (2007) 1 [arXiv:0706. 0654] [INSPIRE].

[10] G. Breit and J.A. Wheeler, Collision of two light quanta, Phys. Rev. 46 (1934) 1087 [INSPIRE].

[11] L.D. Landau and E.M. Lifshitz, On the production of electrons and positrons by a collision of two particles, Phys. Z. Sowjet. 6 (1934) 244 [INSPIRE].

[12] G. Racah, Sulla Nascita di Coppie per Urti di Particelle Elet-trizzate, Nuovo Cim. 14 (1937) 93 [INSPIRE].

[13] H.D. Politzer, Reliable Perturbative Results for Strong Interactions?, Phys. Rev. Lett. 30 (1973) 1346 [INSPIRE].

[14] D.J. Gross and F. Wilczek, Ultraviolet Behavior of Nonabelian Gauge Theories, Phys. Rev. Lett. 30 (1973) 1343 [INSPIRE].

[15] H.A. Bethe and L.C. Maximon, Theory of Bremsstrahlung and Pair Production. 1. Differential Cross Section, Phys. Rev. 93 (1954) 768 [INSPIRE].

[16] H. Davies, H.A. Bethe and L.C. Maximon, Theory of Bremsstrahlung and Pair Production. 2. Integral Cross Section for Pair Production, Phys. Rev. 93 (1954) 788 [inSPIRE].

[17] H. Bethe and W. Heitler, On the Stopping of fast particles and on the creation of positive electrons, Proc. Roy. Soc. Lond. A 146 (1934) 83. 
[18] D. Ivanov and K. Melnikov, Lepton pair production by a high-energy photon in a strong electromagnetic field, Phys. Rev. D 57 (1998) 4025 [hep-ph/9709352] [InSPIRE].

[19] A.J. Baltz and L.D. McLerran, Two center light cone calculation of pair production induced by ultrarelativistic heavy ions, Phys. Rev. C 58 (1998) 1679 [nucl-th/9804042] [INSPIRE].

[20] B. Segev and J.C. Wells, Exact $Z^{* * 2}$ scaling of pair production in the high-energy limit of heavy ion collisions, Phys. Rev. C 59 (1999) 2753 [physics/9805013] [InSPIRE].

[21] K. Hencken, D. Trautmann and G. Baur, Calculation of higher order effects in electron positron pair production in relativistic heavy ion collisions, Phys. Rev. C 59 (1999) 841 [nucl-th/9808035] [INSPIRE].

[22] Z.-h. Sun, D.-x. Zheng, J. Zhou and Y.-j. Zhou, Studying Coulomb correction at EIC and EicC, Phys. Lett. B 808 (2020) 135679 [arXiv: 2002.07373] [InSPIRE].

[23] R.N. Lee, A.I. Milstein and V.M. Strakhovenko, High-energy expansion of Coulomb corrections to the $e^{+} e^{-}$photoproduction cross-section, Phys. Rev. A 69 (2004) 022708 [hep-ph/0310108] [INSPIRE].

[24] R.N. Lee and A.I. Milstein, Strong suppression of Coulomb corrections to the cross section of $e^{+} e^{-}$pair production in ultrarelativistic nuclear collisions, Zh. Eksp. Teor. Fiz. 136 (2009) 1121 [arXiv: 0903.0235] [INSPIRE].

[25] A.J. Baltz, Evidence for higher order QED in $e^{+} e^{-}$pair production at RHIC, Phys. Rev. Lett. 100 (2008) 062302 [arXiv:0710.4944] [INSPIRE].

[26] A.J. Baltz and J. Nystrand, Higher order QED in high mass $e^{+} e^{-}$pairs production at RHIC, Phys. Rev. C 82 (2010) 027901 [arXiv: 1003.3864] [InSPIRE].

[27] C.R. Vane et al., Electron positron pair production in Coulomb collisions of ultrarelativistic sulphur ions with fixed targets, Phys. Rev. Lett. 69 (1992) 1911 [INSPIRE].

[28] CERES/NA45 collaboration, Measurement of electromagnetically produced $e^{+} e^{-}$pairs in distant S-Pt collisions, Phys. Lett. B 332 (1994) 471 [INSPIRE].

[29] STAR collaboration, Production of $e^{+} e^{-}$pairs accompanied by nuclear dissociation in ultra-peripheral heavy ion collision, Phys. Rev. C 70 (2004) 031902 [nucl-ex/0404012] [INSPIRE].

[30] PHENIX collaboration, Photoproduction of $J / \psi$ and of high mass $e^{+} e^{-}$in ultra-peripheral Au + Au collisions at $\sqrt{s_{\mathrm{NN}}}=200 \mathrm{GeV}$, Phys. Lett. B 679 (2009) 321 [arXiv:0903.2041] [INSPIRE].

[31] ALICE collaboration, Charmonium and $e^{+} e^{-}$pair photoproduction at mid-rapidity in ultra-peripheral $P b-P b$ collisions at $\sqrt{s_{\mathrm{NN}}}=2.76$ TeV, Eur. Phys. J. C 73 (2013) 2617 [arXiv: 1305.1467] [INSPIRE].

[32] CMS collaboration, Evidence for light-by-light scattering and searches for axion-like particles in ultraperipheral $\mathrm{PbPb}$ collisions at $\sqrt{s_{\mathrm{NN}}}=5.02 \mathrm{TeV}$, Phys. Lett. B 797 (2019) 134826.

[33] ATLAS collaboration, Exclusive dimuon production in ultraperipheral $\mathrm{Pb}+\mathrm{Pb}$ collisions at $\sqrt{s_{\mathrm{NN}}}=5.02 \mathrm{TeV}$ with ATLAS, arXiv:2011.12211 [INSPIRE].

[34] STAR collaboration, Measurement of $e^{+} e^{-}$Momentum and Angular Distributions from Linearly Polarized Photon Collisions, Phys. Rev. Lett. 127 (2021) 052302 [arXiv: 1910.12400] [INSPIRE]. 
[35] M.L. Miller, K. Reygers, S.J. Sanders and P. Steinberg, Glauber modeling in high energy nuclear collisions, Ann. Rev. Nucl. Part. Sci. 57 (2007) 205 [nucl-ex/0701025] [InSPIRE].

[36] R.C. Barrett and D.F. Jackson, Nuclear Sizes and Structure, Oxford University Press (1977) [DOI].

[37] A1 collaboration, High-precision determination of the electric and magnetic form factors of the proton, Phys. Rev. Lett. 105 (2010) 242001 [arXiv:1007.5076] [INSPIRE].

[38] K. Hencken, D. Trautmann and G. Baur, Impact parameter dependence of the total probability for the electromagnetic electron - positron pair production in relativistic heavy ion collisions, Phys. Rev. A 51 (1995) 1874 [nucl-th/9410014] [INSPIRE].

[39] A. Alscher, K. Hencken, D. Trautmann and G. Baur, Multiple electromagnetic electron positron pair production in relativistic heavy ion collisions, Phys. Rev. A 55 (1997) 396 [nucl-th/9606011] [INSPIRE].

[40] V. Shtabovenko, R. Mertig and F. Orellana, New Developments in FeynCalc 9.0, Comput. Phys. Commun. 207 (2016) 432 [arXiv:1601.01167] [INSPIRE].

[41] G.P. Lepage, A New Algorithm for Adaptive Multidimensional Integration, J. Comput. Phys. 27 (1978) 192 [INSPIRE].

[42] S. Klein, A.H. Mueller, B.-W. Xiao and F. Yuan, Acoplanarity of a Lepton Pair to Probe the Electromagnetic Property of Quark Matter, Phys. Rev. Lett. 122 (2019) 132301 [arXiv: 1811.05519] [INSPIRE].

[43] ATLAS collaboration, Observation of centrality-dependent acoplanarity for muon pairs produced via two-photon scattering in $\mathrm{Pb}+\mathrm{Pb}$ collisions at $\sqrt{s_{\mathrm{NN}}}=5.02 \mathrm{TeV}$ with the ATLAS detector, Phys. Rev. Lett. 121 (2018) 212301 [arXiv: 1806.08708] [INSPIRE].

[44] T. Sjöstrand, S. Mrenna and P.Z. Skands, PYTHIA 6.4 Physics and Manual, JHEP 05 (2006) 026 [hep-ph/0603175] [INSPIRE].

[45] T. Sjöstrand et al., An introduction to PYTHIA 8.2, Comput. Phys. Commun. 191 (2015) 159 [arXiv: 1410.3012] [inSPIRE].

[46] D.Y. Ivanov, A. Schiller and V.G. Serbo, Large Coulomb corrections to the $e^{+} e^{-}$pair production at relativistic heavy ion colliders, Phys. Lett. B 454 (1999) 155 [hep-ph/9809449] [INSPIRE].

[47] F. Krauss, M. Greiner and G. Soff, Photon and gluon induced processes in relativistic heavy ion collisions, Prog. Part. Nucl. Phys. 39 (1997) 503 [InSPIRE].

[48] W. Zha, J.D. Brandenburg, Z. Tang and Z. Xu, Initial transverse-momentum broadening of Breit-Wheeler process in relativistic heavy-ion collisions, Phys. Lett. B 800 (2020) 135089.

[49] J. Jastrzebski et al., Neutron density distributions from antiprotonic atoms compared with hadron scattering data, Int. J. Mod. Phys. E 13 (2004) 343 [INSPIRE].

[50] PREX collaboration, Accurate Determination of the Neutron Skin Thickness of ${ }^{208} \mathrm{~Pb}$ through Parity-Violation in Electron Scattering, Phys. Rev. Lett. 126 (2021) 172502 [arXiv: 2102.10767] [INSPIRE].

[51] Particle Data Group collaboration, Review of Particle Physics, PTEP 2020 (2020) 083C01 [INSPIRE]. 
[52] L. Frankfurt, C.E. Hyde, M. Strikman and C. Weiss, Generalized parton distributions and rapidity gap survival in exclusive diffractive pp scattering, Phys. Rev. D 75 (2007) 054009 [hep-ph/0608271] [INSPIRE].

[53] ATLAS collaboration, Measurement of the Inelastic Proton-Proton Cross-Section at $\sqrt{s}=7 \mathrm{TeV}$ with the ATLAS Detector, Nature Commun. 2 (2011) 463.

[54] A.J. Baltz, M.J. Rhoades-Brown and J. Weneser, Heavy ion partial beam lifetimes due to Coulomb induced processes, Phys. Rev. E 54 (1996) 4233 [inSPIRE].

[55] A. Veyssiere, H. Beil, R. Bergere, P. Carlos and A. Lepretre, Photoneutron cross sections of $208 \mathrm{~Pb}$ and 197 Au, Nucl. Phys. A 159 (1970) 561 [inSPIRE].

[56] A. Lepretre et al., Measurements of the Total Photonuclear Cross-sections From $30 \mathrm{MeV}$ to $140 \mathrm{MeV}$ for SN, Ce, Ta, Pb and U Nuclei, Nucl. Phys. A 367 (1981) 237 [InSPIRE].

[57] P. Carlos et al., Total photonuclear absorption cross section for $\mathrm{Pb}$ and for heavy nuclei in the Delta resonance region, Nucl. Phys. A 431 (1984) 573 [INSPIRE].

[58] T.A. Armstrong et al., Total hadronic cross-section of gamma rays in hydrogen in the energy range 0.265-GeV to 4.215-GeV, Phys. Rev. D 5 (1972) 1640 [INSPIRE].

[59] D.O. Caldwell, V.B. Elings, W.P. Hesse, R.J. Morrison, F.V. Murphy and D.E. Yount, Total Hadronic Photoabsorption Cross-Sections on Hydrogen and Complex Nuclei from $4 \mathrm{GeV}$ to $18 \mathrm{GeV}$, Phys. Rev. D 7 (1973) 1362 [inSPIRE].

[60] S. Michalowski et al., Experimental Study of Nuclear Shadowing in Photoproduction, Phys. Rev. Lett. 39 (1977) 737 [InSPIRE].

[61] T.A. Armstrong et al., The total photon deuteron hadronic cross-section in the energy range 0.265-4.215 GeV, Nucl. Phys. B 41 (1972) 445 [inSPIRE].

[62] M. Broz, J.G. Contreras and J.D. Tapia Takaki, A generator of forward neutrons for ultra-peripheral collisions: n00n, Comput. Phys. Commun. 253 (2020) 107181.

[63] G. Baur, K. Hencken and D. Trautmann, Photon-photon physics in very peripheral collisions of relativistic heavy ions, J. Phys. G 24 (1998) 1657 [hep-ph/9804348] [INSPIRE].

[64] CDF collaboration, Observation of exclusive charmonium production and $\gamma+\gamma$ to $\mu^{+} \mu^{-}$in $p \bar{p}$ collisions at $\sqrt{s}=1.96$ TeV, Phys. Rev. Lett. 102 (2009) 242001 [arXiv:0902.1271] [INSPIRE].

[65] CDF collaboration, Observation of Exclusive Electron-Positron Production in Hadron-Hadron Collisions, Phys. Rev. Lett. 98 (2007) 112001 [hep-ex/0611040] [INSPIRE].

[66] ATLAS collaboration, Measurement of exclusive $\gamma \gamma \rightarrow \ell^{+} \ell^{-}$production in proton-proton collisions at $\sqrt{s}=7 \mathrm{TeV}$ with the ATLAS detector, Phys. Lett. B $\mathbf{7 4 9}$ (2015) 242 [arXiv: 1506. 07098] [INSPIRE].

[67] CMS collaboration, Search for Exclusive or Semi-Exclusive Photon Pair Production and Observation of Exclusive and Semi-Exclusive Electron Pair Production in pp Collisions at $\sqrt{s}=7 \mathrm{TeV}$, JHEP 11 (2012) 080 [arXiv:1209.1666] [INSPIRE].

[68] S.R. Klein, J. Nystrand, J. Seger, Y. Gorbunov and J. Butterworth, STARlight: A Monte Carlo simulation program for ultra-peripheral collisions of relativistic ions, Comput. Phys. Commun. 212 (2017) 258 [arXiv: 1607.03838] [INSPIRE]. 